Elect. Comm. in Probab. 16 (2011), 458-466

\title{
THE TAIL OF THE MAXIMUM OF BROWNIAN MOTION MINUS A PARABOLA
}

\author{
PIET GROENEBOOM \\ Delft University of Technology, Mekelweg 4, 2628 CD Delft, The Netherlands, \\ http: //dutiosc. twi. tudelft. nl/ pietg/ \\ email: p.groeneboom@tudelft.nl \\ NICO M. TEMME \\ CWI, Science Park 123, 1098 XG Amsterdam, The Netherlands, \\ http: //homepages. cwi. nl/ nicot/ \\ email: Nico.Temme@cwi.nl
}

Submitted November 15, 2010, accepted in final form July 16, 2011

AMS 2000 Subject classification: 60J65,60J75

Keywords: Brownian motion, parabolic drift, maximum, Airy functions

Abstract

We analyze the tail behavior of the maximum $N$ of $\left\{W(t)-t^{2}: t \geq 0\right\}$, where $W$ is standard Brownian motion on $[0, \infty)$, and give an asymptotic expansion for $\mathbb{P}\{N \geq x\}$, as $x \rightarrow \infty$. This extends a first order result on the tail behavior, which can be deduced from [5]. We also point out the relation between certain results in [3] and [6].

\section{Introduction}

The distribution function of the maximum of Brownian motion minus a parabola was studied in the two recent papers [6] and [3], both for one-sided and two-sided Brownian motion. The characterization of the distribution function is somewhat different in the two papers, but both characterizations (unavoidably) involve Airy functions. In this note we address the tail behavior of the distribution, a topic that was not addressed in these papers.

The tail behavior of the maximum plays an important role in certain recent studies on the asymptotic distribution of tests for monotone hazards, based on integral-type statistics measuring the distance between the empirical cumulative hazard function and its greatest convex minorant, for example in [4].

Let $N$ be defined by

$$
N=\max _{t \geq 0}\left\{W(t)-t^{2}\right\}
$$

where $W$ is standard Brownian motion on $[0, \infty)$. It can be deduced from Theorem 2.1 in [5] that 
the distribution function $F_{N}$ of $N$ satisfies:

$$
1-F_{N}(x) \sim \frac{1}{\sqrt{3}} \exp \left\{-\frac{8 x^{3 / 2}}{3 \sqrt{3}}\right\}, x \rightarrow \infty .
$$

In section 2 we will give an asymptotic expansion of the left-hand side, which extends this result. The proof is based on an integral expression for the density, derived from [3] (which in turn relies on [2]), and uses a saddle point method for the integral over a shifted path in the complex plane. As a side effect, it also leads to a clarification of the relation between the representations of the distribution, given in [6] and [3].

\section{Main results}

In the following, we will use Corollary 2.1 of [3], which is stated below for ease of reference, specialized to the density of the maximum of $W(t)-t^{2}$ (instead of the more general $W(t)-c t^{2}$ ).

Lemma 2.1. (Corollary 2.1 in [3]) For $x>0$ the density $f_{N}$ of $N$ is given by:

$$
f_{N}(x)=2^{2 / 3}\left\{\operatorname{Ai}\left(2^{2 / 3} x\right)-2 \operatorname{Re}\left(e^{-i \pi / 6} \int_{0}^{\infty} \frac{\operatorname{Ai}\left(e^{-i \pi / 6} u\right) \operatorname{Ai}^{\prime}\left(i u+2^{2 / 3} x\right)}{\operatorname{Ai}(i u)} d u\right)\right\},
$$

where Ai is the Airy function Ai, as defined in, e.g., [9] ${ }^{1}$.

We deduce from this the following representation which is better suited for our purposes.

Lemma 2.2. For $x>0$ the density $f_{N}$ of $N$ is given by:

$$
f_{N}(x)=\frac{2^{2 / 3}}{\pi} \operatorname{Re}\left(\int_{0}^{\infty} \frac{\operatorname{Ai}\left(i u+2^{2 / 3} x\right)}{\operatorname{Ai}(i u)^{2}} d u\right)=\frac{1}{2^{1 / 3} \pi} \int_{-\infty}^{\infty} \frac{\operatorname{Ai}\left(i u+2^{2 / 3} x\right)}{\operatorname{Ai}(i u)^{2}} d u .
$$

Proof. Integration by parts of the second term of (2.2) yields:

$$
f_{N}(x)=2 \cdot 2^{2 / 3} \operatorname{Re}\left(\int_{0}^{\infty} \operatorname{Ai}\left(i u+2^{2 / 3} x\right) \frac{d}{d u}\left\{\frac{e^{-i \pi / 6} \operatorname{Ai}\left(e^{-i \pi / 6} u\right)}{i \operatorname{Ai}(i u)}\right\} d u\right) .
$$

Let the function $h$ be defined by

$$
h(u)=\frac{d}{d u}\left\{\frac{e^{-i \pi / 6} \operatorname{Ai}\left(e^{-i \pi / 6} u\right)}{i \operatorname{Ai}(i u)}\right\} .
$$

Using $[9]^{2}$ :

$$
\operatorname{Ai}\left(e^{-i \pi / 6} u\right)=\operatorname{Ai}\left(e^{-2 i \pi / 3} i u\right)=\frac{1}{2} e^{-i \pi / 3}\{\operatorname{Ai}(i u)+i \operatorname{Bi}(i u)\}
$$

we obtain

$$
h(u)=\frac{1}{2}\left\{\frac{\mathrm{Ai}^{\prime}(i u)+i \mathrm{Bi}^{\prime}(i u)}{i \operatorname{Ai}(i u)}-\frac{(\mathrm{Ai}(i u)+i \operatorname{Bi}(i u)) \mathrm{Ai}^{\prime}(i u)}{i \operatorname{Ai}(i u)^{2}}\right\},
$$

\footnotetext{
${ }^{1}$ http://dlmf.nist.gov/9

${ }^{2}$ http://dlmf.nist.gov/9.2.E11
} 
and using the Wronskian $\operatorname{Ai}(z) \operatorname{Bi}^{\prime}(z)-\operatorname{Ai}^{\prime}(z) \operatorname{Bi}(z)=1 / \pi$ we conclude that

$$
h(u)=\frac{1}{2 \pi \mathrm{Ai}(i u)^{2}} \text {. }
$$

This gives the desired result.

Remark 2.1. Lemma 2.2 is in fact equivalent to relation (5.10) in [6]. The difference in the scaling constants is caused by the fact that they consider the maximum of $W(t)-\frac{1}{2} t^{2}$ instead of the maximum of $W(t)-t^{2}$ (see also section 3) and the fact that they integrate from $-\infty$ to $\infty$ (in that way also the imaginary part drops out). However, they arrive at this relation in a completely different way. So in this case we can go from Corollary 2.1 in [3] to the result in [6], just by using integration by parts. This might serve as a first step in establishing the relation between the representations in the two papers.

We are now ready to prove our main result.

Theorem 2.1. Let $N$ be defined by (1.1), and let $f_{N}$ and $F_{N}$ be the density and the distribution function of $N$, respectively. Then,

(i)

$$
f_{N}(x) \sim \frac{4 \sqrt{x}}{3} \exp \left(-\frac{8 x^{3 / 2}}{3 \sqrt{3}}\right) \sum_{k=0}^{\infty} \frac{b_{k}}{x^{3 k / 2}}, \quad x \rightarrow \infty,
$$

where the first coefficients are

$$
b_{0}=1, \quad b_{1}=\frac{19}{48} \sqrt{3}, \quad b_{2}=-\frac{3851}{1536}, \quad b_{3}=\frac{3380005}{221184} \sqrt{3}, \quad b_{4}=-\frac{6474441455}{14155776} .
$$

(ii)

$$
1-F_{N}(x) \sim \frac{1}{\sqrt{3}} \exp \left(-\frac{8 x^{3 / 2}}{3 \sqrt{3}}\right) \sum_{k=0}^{\infty} \frac{c_{k}}{x^{3 k / 2}}, \quad x \rightarrow \infty,
$$

where the first coefficients are

$$
c_{0}=1, \quad c_{1}=\frac{19}{48} \sqrt{3}, \quad c_{2}=-\frac{4535}{1536}, \quad c_{3}=\frac{3869785}{221184} \sqrt{3}, \quad c_{4}=-\frac{7310315015}{14155776} .
$$

Proof. Here we only derive the leading terms. Further terms in the asymptotic expansion of $f_{N}(x)$ are computed in the appendix, and those for $F_{N}(x)$ follow upon integrating the expansion for $f_{N}(x)$.

We start with the second representation in (2.3), and write for $x>0$

$$
f_{N}(x)=\frac{1}{2^{1 / 3} \pi} \int_{-\infty}^{\infty} \frac{\operatorname{Ai}\left(i u+2^{2 / 3} x\right)}{\operatorname{Ai}(i u)^{2}} d u=\frac{x}{2^{1 / 3} \pi i} \int_{-i \infty}^{i \infty} \frac{\operatorname{Ai}\left(x\left(u+2^{2 / 3}\right)\right)}{\operatorname{Ai}(x u)^{2}} d u .
$$

We need the well-known asymptotic behavior of the Airy function $\left(\right.$ see $\left.[9]^{3}\right)$ :

$$
\operatorname{Ai}(z) \sim \frac{e^{-\zeta}}{2 \sqrt{\pi} z^{1 / 4}}, \quad \zeta=\frac{2}{3} z^{3 / 2}, z \rightarrow \infty, \quad|\mathrm{ph} z|<\pi .
$$

\footnotetext{
${ }^{3}$ http://dlmf.nist.gov/9.7.E5
} 
It follows that the behavior of the ratio of the Airy functions is given by

$$
\frac{\operatorname{Ai}\left(x\left(u+2^{2 / 3}\right)\right)}{\operatorname{Ai}(x u)^{2}} \sim \frac{2 \sqrt{\pi} x^{1 / 4} u^{1 / 2}}{\left(u+2^{2 / 3}\right)^{1 / 4}} \exp \left(\frac{2}{3} x^{3 / 2} \phi(u)\right), \quad x u \rightarrow \infty, \quad|\operatorname{ph} x u|<\pi,
$$

where

$$
\phi(u)=2 u^{3 / 2}-\left(u+2^{2 / 3}\right)^{3 / 2} .
$$

This function has a minimum at $u=c=\frac{1}{3} 2^{2 / 3}$, and we shift the path of integration in the second integral in (2.5) to the path $\mathscr{P}$ parallel to the imaginary axis, and running from $c-i \infty$ to $c+i \infty$. A similar path was used in the proof of (ii) of Corollary 3.4 in [2].

A local expansion at $u=c$ gives

$$
\phi(u)=\phi(c)+\frac{1}{2} \phi^{\prime \prime}(c)(u-c)^{2}+\mathscr{O}\left((u-c)^{3}\right),
$$

where

$$
\phi(c)=-\frac{4}{\sqrt{3}}, \quad \phi^{\prime \prime}(c)=\frac{9 \sqrt{3}}{2^{10 / 3}} .
$$

We find a first approximation of $f_{N}(x)$ by neglecting the $\mathscr{O}$-term in the local expansion of $\phi(u)$ and by taking $u=c$ in the factor in front of the exponential factor in (2.7). This gives

$$
\begin{aligned}
f_{N}(x) \sim \frac{x}{2^{1 / 3} \pi i} & \frac{2 \sqrt{\pi} x^{1 / 4} c^{1 / 2}}{\left(c+2^{2 / 3}\right)^{1 / 4}} \exp \left(\frac{2}{3} x^{3 / 2} \phi(c)\right) \times \\
& \int_{c-i \infty}^{c+i \infty} \exp \left(\frac{1}{3} x^{3 / 2} \phi^{\prime \prime}(c)(u-c)^{2}\right) d u .
\end{aligned}
$$

Evaluating the integral:

$$
\int_{c-i \infty}^{c+i \infty} \exp \left(\frac{1}{3} x^{3 / 2} \phi^{\prime \prime}(c)(u-c)^{2}\right) d u=\frac{i \sqrt{\pi} 2^{5 / 3}}{3^{3 / 4} x^{3 / 4}}
$$

we find the requested result

$$
f_{N}(x) \sim \frac{4 \sqrt{x}}{3} \exp \left(-\frac{8 x^{3 / 2}}{3 \sqrt{3}}\right), \quad x \rightarrow \infty .
$$

Upon integrating we obtain the result for the distribution function $F_{N}(x)$.

For two-sided Brownian motion we get similarly:

Corollary 2.1. Let $M$ be defined by

$$
M=\max _{t \in \mathbb{R}}\left\{W(t)-t^{2}\right\}
$$

where $W$ is standard two-sided Brownian motion, originating from zero. and let $f_{M}$ and $F_{M}$ be the density and the distribution function of $M$, respectively. Then:

(i)

$$
f_{M}(x)=2 f_{N}(x) F_{N}(x) \sim \frac{8 \sqrt{x}}{3} \exp \left\{-\frac{8 x^{3 / 2}}{3 \sqrt{3}}\right\}, \quad x \rightarrow \infty
$$


(ii)

$$
1-F_{M}(x) \sim \frac{2}{\sqrt{3}} \exp \left\{-\frac{8 x^{3 / 2}}{3 \sqrt{3}}\right\}, \quad x \rightarrow \infty .
$$

Proof. This follows from Corollary 2.2 of [3], which gives the representation:

$$
f_{M}(x)=2 f_{N}(x) F_{N}(x), \quad x \geq 0 .
$$

\section{Concluding remarks}

As pointed out to us by Svante Janson, the result implies certain facts for the moments of the distribution. For example, applying Theorem 4.5 in [7] together with Theorem 2.1 of the present paper gives:

$$
\left(E M^{r}\right)^{1 / r} \sim \frac{1}{2}(3 / 2)^{1 / 3}(r / e)^{2 / 3}, \quad r \rightarrow \infty .
$$

Theorem 2.1 can also easily be extended to a result for

$$
M_{c}=\max _{t \geq 0}\left\{W(t)-c t^{2}\right\},
$$

by using the scaling relation:

$$
M_{c}=c^{-1 / 3} M,
$$

see (1.7) of [6]. So, for example, Theorem 2.1 implies:

$$
\mathbb{P}\left\{M_{c} \geq x\right\}=\mathbb{P}\left\{M \geq c^{1 / 3} x\right\} \sim \frac{1}{\sqrt{3}} \exp \left\{-\frac{8 x^{3 / 2} \sqrt{c}}{3 \sqrt{3}}\right\}, \quad x \rightarrow \infty .
$$

Also, by Lemma 2.2, the density of $M_{c}$ is given by:

$$
f_{M_{c}}(x)=\frac{(4 c)^{1 / 3}}{\pi} \operatorname{Re}\left(\int_{0}^{\infty} \frac{\operatorname{Ai}\left(i u+(4 c)^{1 / 3} x\right)}{\operatorname{Ai}(i u)^{2}} d u\right), \quad x>0 .
$$

\section{Appendix. Computing more coefficients of the asymptotic expansions}

For obtaining more coefficients in the asymptotic expansion of $f_{N}(x)$ and $F_{N}(x)$ we need more details of the asymptotic behavior of the Airy function. We have the well-known expansion (see $\left.[9]^{4}\right)$ :

$$
\operatorname{Ai}(z) \sim \frac{e^{-\zeta}}{2 \sqrt{\pi} z^{1 / 4}} \sum_{k=0}^{\infty}(-1)^{k} \frac{u_{k}}{\zeta^{k}}, \quad z \rightarrow \infty, \quad|\operatorname{ph} z|<\pi,
$$

where

$$
\zeta=\frac{2}{3} z^{3 / 2}
$$

\footnotetext{
${ }^{4}$ http://dlmf.nist.gov/9.7.E5
} 
and

$$
u_{k}=\frac{(2 k+1)(2 k+3)(2 k+5) \cdots(6 k-1)}{(216)^{k} k !}=\frac{\Gamma\left(3 k+\frac{1}{2}\right)}{54^{k} k ! \Gamma\left(k+\frac{1}{2}\right)},
$$

The first coefficients are

$$
u_{0}=1, \quad u_{1}=\frac{5}{72}, \quad u_{2}=\frac{385}{10368}, \quad u_{3}=\frac{85085}{2239488}, \quad u_{4}=\frac{37182145}{644972544} .
$$

Using (2.7) we write the second integral representation of $f_{N}(x)$ in (2.5) in the form

$$
f_{N}(x)=\frac{2^{2 / 3} x^{5 / 4}}{\sqrt{\pi} i}\left(\frac{c}{4}\right)^{1 / 4} \int_{c-i \infty}^{c+i \infty} \exp \left(\frac{2}{3} x^{3 / 2} \phi(u)\right) A(u, x) d u, \quad c=\frac{1}{3} 2^{2 / 3},
$$

where $A(u, x)$ is a slowly varying function along the path of integration. We have the asymptotic representation

$$
A(u, x) \sim\left(\frac{c}{4}\right)^{-1 / 4} \frac{u^{1 / 2}}{\left(u+2^{2 / 3}\right)^{1 / 4}} \frac{\sum_{k=0}^{\infty}(-1)^{k} \frac{u_{k}}{\zeta^{k}}}{\left(\sum_{k=0}^{\infty}(-1)^{k} \frac{u_{k}}{\eta^{k}}\right)^{2}},
$$

where

$$
\zeta=\xi\left(u+2^{2 / 3}\right)^{3 / 2}, \quad \eta=\xi u^{3 / 2}, \quad \xi=\frac{2}{3} x^{3 / 2}
$$

The path of integration in (4.12) cuts the real $u$-axis at the saddle point of the exponential function. For large $u$ we have $\phi(u) \sim u^{3 / 2}$, and for $u \rightarrow \pm i \infty$ we have ph $\phi(u) \sim \pm 3 \pi / 4$. This shows the rate of convergence along this path. The optimal rate of convergence occurs when we take the saddle point contour, or path of steepest descent, defined by $\mathfrak{I} \phi(u)=\mathfrak{I} \phi(c)=0$. At infinity along this path we have ph $u= \pm 2 \pi / 3$. Along the saddle point contour the quantities $\eta$ and $\zeta$ are large, because the variable of integration is bounded away from the origin. Also, $\eta$ and $\zeta$ have suitable phases for using the asymptotic expansions as shown in (4.13).

By manipulating the asymptotic series, we obtain the following expansion:

$$
A(u, x) \sim\left(\frac{c}{4}\right)^{-1 / 4} \frac{u^{1 / 2}}{\left(u+2^{2 / 3}\right)^{1 / 4}} \sum_{k=0}^{\infty} \frac{A_{k}(u)}{\xi^{k}}
$$

where the first few coefficients are given by

$$
\begin{aligned}
& A_{0}(u)=1 \\
& A_{1}(u)=\frac{-5 R+10}{72 u^{3 / 2}} \\
& A_{2}(u)=\frac{385 R^{2}-620-100 R}{10368 u^{3}}, \\
& A_{3}(u)=\frac{9300 R+138520-85085 R^{3}+11550 R^{2}}{2239488 u^{9 / 2}}, \\
& A_{4}(u)=\frac{-2770400 R-62797040+37182145 R^{4}-1432200 R^{2}-3403400 R^{3}}{644972544 u^{6}},
\end{aligned}
$$

where

$$
R=\frac{u^{3 / 2}}{(u+3 c)^{3 / 2}}
$$


Substituting the expansion in (4.15) into (4.12) we obtain

$$
f_{N}(x) \sim \frac{2^{2 / 3} x^{5 / 4}}{\sqrt{\pi} i}\left(\frac{c}{4}\right)^{1 / 4} \sum_{k=0}^{\infty} \frac{\Phi_{k}(\xi)}{\xi^{k}}
$$

where

$$
\Phi_{k}(\xi)=\left(\frac{c}{4}\right)^{-1 / 4} \int_{\infty e^{-2 \pi i / 3}}^{\infty e^{2 \pi i / 3}} \frac{u^{1 / 2}}{\left(u+2^{2 / 3}\right)^{1 / 4}} \exp (\xi \phi(u)) A_{k}(u) d u .
$$

Next we transform these integrals into a standard form by putting

$$
\phi(u)-\phi(c)=\frac{1}{2} \phi^{\prime \prime}(c) v^{2},
$$

where $\phi(c)$ and $\phi^{\prime \prime}(c)$ are given in (2.8). We prescribe $\operatorname{sign}(u-c)=\operatorname{sign}(v)$, and we need the coefficients in the expansion

$$
u=c+v+d_{2} v^{2}+d_{3} v^{3}+\ldots,
$$

of which the first few are given by

$$
d_{2}=\frac{5}{48 c}, \quad d_{3}=-\frac{1}{72 c^{2}}, \quad d_{4}=\frac{115}{27648 c^{3}}, \quad d_{5}=-\frac{385}{221184 c^{4}}, \quad d_{6}=\frac{1705}{1990656 c^{5}} .
$$

The transformation gives

$$
\Phi_{k}(u)=\exp (\xi \phi(c)) \int_{-i \infty}^{i \infty} B_{k}(v) \exp \left(\frac{1}{2} \phi^{\prime \prime}(c) \xi v^{2}\right) d v,
$$

where

$$
B_{k}(v)=\left(\frac{c}{4}\right)^{-1 / 4} \frac{u^{1 / 2}}{(u+3 c)^{1 / 4}} A_{k}(u) \frac{d u}{d v} .
$$

The final step is to expand each $B_{k}(v)$ :

$$
B_{k}(v)=\sum_{j=0}^{\infty} B_{j}^{(k)} v^{j}
$$

to obtain the expansions for $\Phi_{k}(\xi)$ :

$$
\Phi_{k}(\xi) \sim i \sqrt{\frac{2 \pi}{\phi^{\prime \prime}(c) \xi}} \exp (\xi \phi(c)) \sum_{j=0}^{\infty} \frac{C_{j}^{(k)}}{\xi^{j}}, \quad C_{j}^{(k)}=\frac{B_{2 j}^{(k)}(-1)^{j} 2^{j}\left(\frac{1}{2}\right)_{j}}{\left(\phi^{\prime \prime}(c)\right)^{j}},
$$

and putting these expansions into (4.17). This gives

$$
f_{N}(x) \sim \frac{4 \sqrt{x}}{3} \exp \left(-\frac{8 x^{3 / 2}}{3 \sqrt{3}}\right) \sum_{k=0}^{\infty} \frac{D_{k}}{\xi^{k}}, \quad D_{k}=\sum_{j=0}^{k} C_{j}^{(k-j)},
$$

and the $b_{k}$ of (2.4) are given by

$$
b_{k}=\left(\frac{3}{2}\right)^{k} D_{k}, \quad k=0,1,2, \ldots
$$


Table 1: The first coefficients $C_{j}^{(k)}, j, k=0,1,2,3,4$, defined in (4.22).

\begin{tabular}{cccc}
$k$ & $C_{0}^{(k)}$ & $C_{1}^{(k)}$ & $C_{2}^{(k)}$ \\
\hline 0 & 1 & $\frac{79}{1152} \sqrt{3}$ & $-\frac{100031}{884736}$ \\
1 & $\frac{25}{128} \sqrt{3}$ & $-\frac{29165}{49152}$ & $\frac{180113185}{113246208} \sqrt{3}$ \\
2 & $-\frac{13365}{32768}$ & $\frac{8404125}{4194304} \sqrt{3}$ & $-\frac{40293630095}{1073741824}$ \\
3 & $\frac{2649065}{4194304} \sqrt{3}$ & $-\frac{36271635085}{1610612736}$ & $\frac{945966020191985}{3710851743744} \sqrt{3}$ \\
4 & $-\frac{9582104685}{2147483648}$ & $\frac{80522162743295}{824633720832} \sqrt{3}$ & $-\frac{3320764894803103375}{633318697598976}$ \\
\hline$k$ & $C_{3}^{(k)}$ & & $C_{4}^{(k)}$ \\
\hline 0 & $\frac{923668975}{3057647616} \sqrt{3}$ & & $-\frac{18376891706495}{4696546738176}$ \\
1 & $-\frac{2860100405525}{130459631616}$ & & $\frac{85377203586646825}{601157982486528} \sqrt{3}$ \\
2 & $\frac{368687253142325}{1236950581248} \sqrt{3}$ & & $-\frac{49545968934273638575}{5699868278390784}$ \\
3 & $-\frac{40394551713291361525}{4274901208793088}$ & & $-\frac{1753497345653280774030496688175}{3361919107433565782016}$ \\
4 & $\frac{204107643936013882342175}{2188749418902061056} \sqrt{3}$ &
\end{tabular}

In Table 1 we give the first values of $C_{j}^{(k)}$ from which the coefficients $D_{k}$ and $b_{k}$ can be computed.

Acknowledgements We thank the referees for their careful reading and useful comments. We also want to thank Svante Janson and Jon Wellner for their useful comments and Zakhar Kabluchko for bringing [5] to our attention. Nico M. Temme acknowledges financial support from Ministerio de Educación y Ciencia, project MTM2006-09050, Ministerio de Ciencia e Innovación, project MTM2009-11686, and Gobierno of Navarra, Res. 07/05/2008.

\section{References}

[1] P. G. Drazin and W.H. Reid, Hydrodynamic stability, Cambridge University Press, Cambridge, 1981, Cambridge Monographs on Mechanics and Applied Mathematics. MR0604359

[2] P. Groeneboom, Brownian motion with a parabolic drift and Airy functions, Probab. Theory Related Fields 81 (1989), no. 1, 79-109. MR0981568

[3] _ The maximum of Brownian motion minus a parabola, Electron. J. Probab. 15 (2010), no. 62, 1930-1937. MR2738343

[4] P. Groeneboom and G. Jongbloed, Testing monotonicity of a hazard: asymptotic distribution theory., Submitted, 2010.

[5] J. Hüsler and V. Piterbarg, Extremes of a certain class of Gaussian processes, Stochastic Process. Appl. 83 (1999), no. 2, 257-271. MR1708208 
[6] G. Janson, S. Louchard and A. Martin-Löf, The maximum of Brownian motion with parabolic drift, Electron. J. Probab. 15 (2010), no. 61, 1893-1929. MR2738342

[7] S. Janson and P. Chassaing, The center of mass of the ISE and the Wiener index of trees, Electron. Comm. Probab. 9 (2004), 178-187 (electronic). MR2108865

[8] A. B. Olde Daalhuis, Hypergeometric function, NIST handbook of mathematical functions, U.S. Dept. Commerce, Washington, DC, 2010, pp. 383-401. MR2655355

[9] F. W. J. Olver, Airy and related functions, NIST handbook of mathematical functions, U.S. Dept. Commerce, Washington, DC, 2010, pp. 193-213. MR2655349

[10] F. W. J. Olver, D. W. Lozier, R. F. Boisvert, and C. W. Clark (eds.), NIST handbook of mathematical functions, U.S. Department of Commerce National Institute of Standards and Technology, Washington, DC, 2010, With 1 CD-ROM (Windows, Macintosh and UNIX). MR2723248 